\title{
RELATIVE WEAK MIXING IS GENERIC
}

\author{
ELI GLASNER AND BENJAMIN WEISS
}

\begin{abstract}
A classical result of Halmos asserts that among measure preserving transformations the weak mixing property is generic. We extend Halmos' result to the collection of ergodic extensions of a fixed, but arbitrary, aperiodic transformation $T_{0}$. We then use a result of Ornstein and Weiss to extend this relative theorem to the general (countable) amenable group.
\end{abstract}

\section{INTRODUCTION}

In chapter 19 of Halmos' seminal book [Ha-56], entitled "Category", he proves what he calls the "second category theorem" (the original proof was published in [Ha-44]):

0.1. Theorem. In the weak topology the set of all weakly mixing transformations is an everywhere dense $G_{\delta}$.

Only later researchers in ergodic theory got interested in properties of extensions of measure preserving transformations and their structure (see e.g. [Th-75], [Z-76a, Z-76b] and [Fur-77], [Ru-79]). Surprisingly, as far as we know, the natural question, whether Halmos' theorem holds for extensions, did not receive any consideration. The recent result of M. Schnurr [Sch-17] (following a question of Tao) caught our attention and in the present note we extend Halmos' result to the collection of measure preserving transformations which are extensions of a fixed, but arbitrary, aperiodic transformation $T_{0}$.

Let $\nu$ denote the Lebesgue measure on the unit interval $I$. Let Aut $(\nu)$ denote the Polish group of measure preserving automorphisms of the standard Lebesgue space $\left(I, \mathcal{B}_{0}, \nu\right)$. We let $\mu=\nu \times \nu$ be the product measure on the space $X=I \times I$. Sometimes we also write $(X, \mathcal{B}, \mu)=\left(Z, \mathcal{B}_{0}, \nu\right) \times\left(Y, \mathcal{B}_{1}, \nu\right)$, where $Z=I=Y$ and $\mathcal{B}$ and $\mathcal{B}_{1}$ are the corresponding algebras of measurable sets.

Date: July 4, 2018.

Key words and phrases. relative weak mixing, Rohlin tower, amenable groups.

2010 Mathematical Subject Classification: 37A25, 37A05, 37A15, 37A20. 
Let $T_{0} \in \operatorname{Aut}(\nu)$ be a fixed aperiodic transformation. On $(X, \mathcal{B}, \mu)$ let $\mathcal{A}$ denote the $\sigma$-algebra of sets of the form $A \times I, A \in \mathcal{B}_{0}$. Let

$$
\mathcal{M}=\left\{T \in \operatorname{Aut}(\mu): T(A \times I)=T_{0} A \times I \text { for all } A \in \mathcal{B}_{0}\right\}
$$

a closed subset of the Polish group Aut $(\mu)$. Denote by $G$ the closed subgroup of Aut $(\mu)$ consisting of transformations $S \in \operatorname{Aut}(\mu)$ such that $S \uparrow \mathcal{A}=\mathrm{Id}$. Clearly $\mathcal{M}$ is invariant under conjugation by elements of $G$.

Let

$$
X \underset{Z}{\times} X=\left\{\left((z, y),\left(z, y^{\prime}\right)\right): z, y, y^{\prime} \in I\right\}
$$

and let

$$
\lambda=\int_{z \in I}\left(\delta_{z} \times \nu\right) \times\left(\delta_{z} \times \nu\right) d \nu(z)
$$

denote the relatively independent product measure $\underset{Z}{\times} \mu$, which is supported on $X \underset{Z}{X} X$.

0.2. Definition. An element $T \in \mathcal{M}$ is relatively weakly mixing over $T_{0}$ when the measure $\lambda$ is $T \times T$-ergodic.

We can now state our main theorem.

0.3. Theorem. The collection $\mathcal{W}$ of measure preserving transformations in $\mathcal{M}$, which are weakly mixing relative to $T_{0}$, is a dense $G_{\delta}$ subset of $\mathcal{M}$.

In section 1 we prove Theorem 0.3. In section 2 we apply a general argument regarding extensions, originated in [RW-00], and then use a result of Ornstein and Weiss [OW-80] (for proofs see [CFW-81]), to extend the relative theorem to the general (countable) amenable group.

We thank J.-P. Thouvenot and Michael Schnurr for suggesting the present formulations of Proposition 1.3 and Theorem 0.3, which are now slightly stronger than the original ones (see [GW-18]).

\section{The RELATIVE THEOREM FOR $\mathbb{Z}$-ACTIONS}

We first note that, by a theorem of Rohlin, we can write every $T \in \mathcal{M}$ as a skew product over $T_{0}$ :

$$
T(z, y)=\left(T_{0} z, \tau_{z}(y)\right)
$$

where $z \mapsto \tau_{z}$ is a measurable map from $Z$ into the Polish group Aut $(\nu)$ (see e.g. [Gl-03, Theorem 3.18]). 
1.1. Definition. Given a measurable partition $\left\{A_{0}, A_{1}, \ldots, A_{k}\right\}$ of $I$ and a finite set $\left\{R_{0}, R_{1}, \ldots, R_{k}\right\}$ of elements of Aut $(\nu)$, define a piecewise constant skew product over $T_{0}, R \in \mathcal{M} \subset$ Aut $(\mu)$ by the formula

$$
R(z, y)=\left(T_{0} z, \rho_{z}(y)\right)
$$

where for each $j, \rho_{z}$ is the constant transformation $R_{j}$ on the cell $A_{j}$.

1.2. Lemma. The collection of piecewise constant skew products over $T_{0}$ is a dense subset of $\mathcal{M}$.

Proof. Let $d$ be a compatible metric on Aut $(\nu)$. An arbitrary element $T \in \mathcal{M}$ has the form

$$
T(z, y)=\left(T_{0} z, \tau_{z} y\right)
$$

where $\tau:[0,1] \rightarrow \operatorname{Aut}(\nu)$ is a measurable mapping. Denote by $\tilde{\nu}$ the push-forward measure $\tilde{\nu}=\tau_{*} \nu=\nu \circ \tau^{-1}$. This is a regular measure on Aut $(\nu)$ hence, given $\epsilon>0$, there is a compact set $K \subset \operatorname{Aut}(\nu)$ with $\tilde{\nu}(K)>1-\epsilon$. Let $\left\{C_{i}\right\}_{i=1}^{k}$ be a partition of $K$ into sets with diameter $<\epsilon$ and set $C_{0}=\operatorname{Aut}(\nu) \backslash K$. Let $A_{i}=\tau^{-1}\left(C_{i}\right), 0 \leq i \leq k$. Define $R_{0}=$ Id and for each $1 \leq i \leq k$ choose some $R_{i} \in C_{i}$. Finally let $R=R_{\left\{A_{i}, R_{i}\right\}}$ be defined by

$$
R(z, y)=\left(T_{0} z, \rho_{z}(y)\right)
$$

where for each $i, \rho_{z}$ is the constant transformation $R_{i}$ on the cell $A_{i}$. Clearly $T \stackrel{\epsilon}{\sim}$ $R$.

We can now prove a relative analogue of Halmos' conjugation theorem for aperiodic transformations:

1.3. Proposition. For every element $T \in \mathcal{M}$, its orbit under conjugations with elements of $G$ is dense in $\mathcal{M}$.

Proof. We write $T$ as a skew product over $T_{0}$ :

$$
T(z, y)=\left(T_{0} z, \tau_{z}(y)\right)
$$

By Lemma 1.2 it suffices to show that given $R=R_{\left\{A_{j}, R_{j}\right\}}$, a piecewise constant skew product over $T_{0}$, and $\epsilon>0$, there is some $Q \in G$ such that, with $V=Q T Q^{-1}$ we have $V \stackrel{\epsilon}{\sim} R$.

Fix $n$ so that $\frac{1}{n}<\epsilon / 2$. Let $\left\{B, T_{0} B, \ldots, T^{n-1} B\right\}$ be a Rohlin tower for $T_{0}$ with $\nu\left(I \backslash \bigcup_{i=0}^{n-1} T_{0}^{i} B\right)<\epsilon / 2$ (see e.g. $[\mathrm{Ha}-56]$ ). 
We refine the tower with respect to the partition $\left\{A_{j}: 1 \leq j \leq k\right\}$. This partitions $B$ into subsets $\left\{B_{l}: 1 \leq l \leq L\right\}$, such that for each $l$ and each $i$ there is some $\alpha(l, i)$ with $T^{i} B_{l} \subset A_{\alpha(l, i)}$.

Next, define $Q \in G$,

$$
Q(z, y)=\left(z, \kappa_{z}(y)\right)
$$

as follows:

- For each $l$, set $\kappa^{(l)} z=\operatorname{Id}$ on $B_{l}$,

- for $i=1, \ldots, n-2$, let $\kappa_{z}^{(l)} \in \operatorname{Aut}(\nu)$ satisfy $\kappa^{(l)} T_{0} z \tau_{z}\left(\kappa^{(l)} z\right)^{-1}=R_{\alpha(l, i)}$, for $z \in T_{0}^{i} B_{l}, i=0,1, \ldots, n-2$,

- $\kappa^{(l)} T_{0} z \tau_{z}\left(\kappa^{(l)}\right)^{-1}=R_{0}$ for $z \in T_{0}^{n-1} B_{l}$.

Thus

$$
\begin{gathered}
\kappa_{z}^{(l)}=\mathrm{Id}, \quad z \in B_{l} \\
\kappa_{T_{0}^{i+1} z}^{(l)}=R_{\alpha(l, i)} \kappa^{(l)} T_{0}^{i z}\left(\tau_{T_{0}^{i} z}\right)^{-1}, \quad z \in B_{l}, i=1, \ldots n-1,
\end{gathered}
$$

so that $Q$ is defined by

$$
\begin{gathered}
Q(z, y)=\left(z, \kappa_{z}^{-1} y\right) \quad \text { for } \quad z \in T_{0}^{i} B_{l}, \quad 0 \leq i \leq n-1, \quad 1 \leq l \leq L \\
Q(z, y)=(z, y), \quad \text { for } \quad z \in I \backslash \bigcup_{i=0}^{n-1} T_{0}^{i} B
\end{gathered}
$$

Set $V=Q T Q^{-1}$. It is now easy to check that $V \stackrel{\epsilon}{\sim} R$, and we are done.

1.4. Lemma. The collection $\mathcal{W}$ consisting of transformations which are relatively weakly mixing over $T_{0}$ is a $G_{\delta}$ subset of $\mathcal{M}$.

Proof. Given $A, B \in X$, the $\sigma$-algebra of measurable subsets on $X, \epsilon>0$ and $N \in \mathbb{N}$, set

$\mathcal{E}_{A, B, \epsilon, N}=\left\{T \in \mathcal{M}:\left\|\frac{1}{N} \sum_{n=0}^{N-1}\left(T^{n} \times T^{n}\right)\left(\mathbf{1}_{A} \otimes \mathbf{1}_{B}\right)-\mathbb{E}\left(\mathbb{E}\left(\mathbf{1}_{A} \mid Z\right) \mathbb{E}\left(\mathbf{1}_{B} \mid Z\right)\right)\right\|_{L^{2}(\lambda)}<\epsilon\right\}$.

Clearly each $\mathcal{E}_{A, B, \epsilon, N}$ is an open set and

$$
\mathcal{W}=\bigcap_{i, j} \bigcap_{k} \bigcup_{N} \mathcal{E}_{A_{i}, A_{j}, \frac{1}{k}, N}
$$

where $\left\{A_{i}\right\}_{i \in \mathbb{N}}$ is a countable dense collection in $X$.

We are now in a position to complete the proof of our main theorem. 
Proof of Theorem 0.3. Let $W_{0} \in \operatorname{Aut}(\nu)$ be a fixed weakly mixing transformation and set $W=T_{0} \times W_{0}$ on $I \times I$. Note that $W$ is a weakly mixing extension of $T_{0}$.

By Proposition 1.3 the $G$-orbit of $W$ is dense in $\mathcal{M}$. Since every element of this orbit is weakly mixing relative to $T_{0}$ (being a conjugate of $W=T_{0} \times W_{0}$ ), and as the collection of all the transformations which are weakly mixing relative to $T_{0}$ is a $G_{\delta}$ subset of $\mathcal{M}$ (by Lemma 1.4), the assertion of the theorem follows.

Let

$$
\mathcal{N}=\{T \in \operatorname{Aut}(\mu): T \text { leaves the } \sigma \text {-algebra } \mathcal{A} \text { invariant }\}
$$

and let

$$
\widetilde{\mathcal{W}}=\left\{T \in \mathcal{N}: T \text { is a weakly mixing extension of } T^{\prime}=T \uparrow \mathcal{A}\right\}
$$

As the collection of ergodic transformations is a dense $G_{\delta}$ subset of Aut $(\nu)$, Theorem 0.3, combined with the Kuratowski-Ulam theorem [Ox-80, Theorem 15.4], immediately yield the following result of M. Schnurr [Sch-17]:

1.5. Corollary. The collection $\widetilde{\mathcal{W}}$ forms a residual subset of $\mathcal{N}$.

\section{The RELATIVE THEOREM FOR AMENABLE GROUPS}

The result of Halmos, that weak mixing is generic in Aut $(\nu)$, can be extended to any countable amenable group as soon as one establishes that orbits under conjugation by $\operatorname{Aut}(\nu)$ of free actions are dense. In turn, this relies on the Rohlin lemma (in fact Halmos' original proof used a weaker version of the lemma which was first stated by Rohlin [Ro-48]). Such a result for amenable groups was established in [OW-80]. It is therefore natural to ask about the analogue of theorem 0.3 for actions of an amenable group. The fact that, for a given amenable group $G$, the collection of the relatively weakly mixing extensions of a fixed action forms a $G_{\delta}$ can be proved just like we proved lemma 1.4. It thus remains to show that this collection is dense. In order to do this we proceed as follows.

2.1. Definition. If $G$ and $H$ are two countable groups acting as measure preserving transformations $\left\{T_{g}\right\}_{g \in G},\left\{S_{h}\right\}_{h \in H}$ on the measure space $(Z, \nu)$ we say that the actions are orbit equivalent if for $\nu$-a.e. $z \in Z, G z=H z$.

In [OW-80] and [CFW-81] it is shown that any ergodic measure preserving action of an amenable group is orbit equivalent to an action of $\mathbb{Z}$. 
We fix an arbitrary countable amenable group $G$. As in section 1 we let $\nu$ denote the Lebesgue measure on the unit interval $I$, and let $\mu=\nu \times \nu$ be the product measure on the space $X=I \times I$. Again we write $(X, \mathcal{B}, \mu)=\left(Z, \mathcal{B}_{0}, \nu\right) \times(Y, \mathcal{B}, \nu)$, where $Z=I=Y$.

Let $\mathbb{A}(G, \nu)$ denote the Polish space of measure preserving actions $\left\{T_{g}\right\}_{g \in G}$ of $G$ on $(Z, \nu)$. Let $\left\{\left(T_{0}\right)_{g}\right\}_{g \in G} \in \mathbb{A}(G, \nu)$ be a fixed ergodic $G$-action. On $(X, \mathcal{B}, \mu)$ let $\mathcal{A}(\nu)$ denote the $\sigma$-algebra of sets of the form $A \times I, A \in \mathcal{B}_{0}$. Let

$$
\mathcal{M}=\left\{T \in \mathbb{A}(G, \mu): T_{g}(A \times I)=\left(T_{0}\right)_{g} A \times I \text { for all } A \in \mathcal{B}_{0} \text { and } g \in G\right\},
$$

a closed subset of the Polish space $\mathbb{A}(G, \mu)$.

Next we remark that if two actions $\left\{T_{g}\right\}$ of $G$ and $\left\{S_{h}\right\}$ of $H$ are orbit equivalent, the $T$ action is ergodic if and only if the $S$ action is ergodic. The acting groups may be quite different.

If $S_{0}$ is an action of a group $H$ on $\left(Z, \mathcal{B}_{0}, \nu\right)$ that is orbit equivalent to $T_{0}$, then the collection of $H$-actions $\mathcal{M}^{\prime}$ defined by

$$
\mathcal{M}^{\prime}=\left\{S \in \mathbb{A}(H, \mu): S_{h}(A \times I)=\left(S_{0}\right)_{h} A \times I \text { for all } A \in \mathcal{B}_{0} \text { and } h \in H\right\}
$$

can be matched, via a canonical bijective map, with the collection $\mathcal{M}$ as follows. An element $T \in \mathcal{M}$ is determined uniquely by the cocycle $\tau(z, g)$ with values in Aut $(\nu)$

$$
T_{g}(z, y)=\left(\left(T_{0}\right)_{g} z, \tau(z, g) y\right), \quad g \in G
$$

Such cocycles depend only on the equivalence relation defined on $Z$ by the $G$-action $T_{0}$. Since $S_{0}$ is orbit equivalent to $T_{0}$, i.e. they have the same orbits, each such cocycle also defines a unique element $S \in \mathcal{M}^{\prime}$. Clearly $T$ is weakly mixing over $T_{0}$ iff $S$ is weakly mixing over $S_{0}$.

2.2. Theorem. Let $G$ be a countable amenable group. The collection $\mathcal{W}$ of measure preserving actions in $\mathcal{M}$ which are weakly mixing relative to a given ergodic action $\left\{\left(T_{0}\right)_{g}\right\}_{g \in G}$ is a dense $G_{\delta}$ subset of $\mathcal{M}$.

Proof. Apply the theorem from [OW-80] and [CFW-81] to get a measure preserving transformation $R \in \operatorname{Aut}(\nu)$ that is orbit equivalent to the $G$-action $\left\{\left(T_{0}\right)_{g}\right\}_{g \in G}$. Now check the relative weak mixing using this transformation.

More explicitly, when the equivalence relation is fixed, as above, the extensions of $R$ are in one to one correspondence with cocycles of the $T_{0} G$-action with values in the group of measure preserving transformations of the fiber $\left(Y, \mathcal{B}_{1}, \nu\right)$. Thus theorem 0.3 , when applied to this $\mathbb{Z}$-action, gives the desired result. 


\section{REFERENCES}

[CFW-81] A. Connes, J. Feldman and B. Weiss, An amenable equivalence relation is generated by a single transformation, Ergodic Theory Dynamical Systems, 1 (1981), no. 4, 431-450 (1982).

[Fur-77] H. Furstenberg, Ergodic behavior of diagonal measures and a theorem of Szemerédi on arithmetic progressions, J. d'Analyse Math. 31, (1977), 204-256.

[GW-18] E. Glasner and B. Weiss, Relative weak mixing is generic, arxiv.org/abs/1707.06425.

[Gl-03] E. Glasner, Ergodic Theory via joinings, Math. Surveys and Monographs, AMS, 101, 2003.

[Ha-44] P. Halmos, In general a measure preserving transformation is mixing, Ann. of Math. (2) 45, (1944). 786-792.

[Ha-56] P. R. Halmos, Lectures on ergodic theory, Publications of the Mathematical Society of Japan, no. 3, The Mathematical Society of Japan 1956.

[OW-80] Ornstein, Donald S.; Weiss, Benjamin Ergodic theory of amenable group actions. I. The Rohlin lemma, Bull. Amer. Math. Soc. (N.S.), 2 (1980), no. 1, 161-164.

[Ox-80] Oxtoby, John C. Measure and category. A survey of the analogies between topological and measure spaces. Second edition. Graduate Texts in Mathematics, 2. Springer-Verlag, New YorkBerlin, 1980.

[Ro-48] V. A. Rohlin, "general" measure-preserving transformation is not mixing, (Russian) Doklady Akad. Nauk SSSR (N.S.) 60, (1948). 349-351.

[Ru-79] D. J. Rudolph, Classifying the isometric extensions of a Bernoulli shift, J. Analyse Math. 34 (1978), 36-60 (1979).

[RW-00] D. J. Rudolph and B. Weiss, Entropy and mixing for amenable group actions, Ann. of Math. (2) 151 (2000), no. 3, 1119-1150.

[Sch-17] M. Schnurr, Generic properties of extensions, arXiv:1704.03709

[Th-75] J.-P. Thouvenot, Quelques propriétés des systèmes dynamiques qui se décomposent en un produit de deux systìes dont l'un est un schéma de Bernoulli, Israel J. of Math. 21, (1975), 177-207.

[Z-76a] R. J. Zimmer, Extensions of ergodic group actions, Illinois J. Math. 20, (1976), 373-409.

[Z-76b] R. J. Zimmer, Ergodic actions with generalized discrete spectrum, Illinois J. Math. 20, (1976), 555-588.

Department of Mathematics, Tel Aviv University, Tel Aviv, Israel

E-mail address: glasner@math.tau.ac.il

Institute of Mathematics, Hebrew University of Jerusalem, Jerusalem, Israel E-mail address: weiss@math.huji.ac.il 